\title{
Robust Algorithm for Automated Source Placement in Near- Infrared Diffuse Imaging
}

\author{
Michael Jermyn $^{1)}$, Brian W. Pogue ${ }^{1)}$, Scott C. Davis ${ }^{1)}$, Subhadra S. Srinivasan ${ }^{1)}$, \& Hamid Dehghani ${ }^{1,2)}$ \\ 1) Thayer School of Engineering, Dartmouth College, Hanover, NH-03755 \\ 2) School of Computer Science, University of Birmingham, Edgbaston, Birmingham, B15 2TT, UK \\ Author e-mail address:Michael.jermyn@dartmouth.edu
}

\begin{abstract}
A surface-shrinking algorithm is demonstrated for automated source position localization one scattering depth into discretized simulation domains, in near-infrared imaging. The algorithm allows users to accurately place source fiber locations with minimal guidance. OCIS codes: (100.0100) Image Processing; (100.3010) Image Reconstruction Techniques
\end{abstract}

\section{Introduction}

The application of standard diffusion modeling to Near-Infrared Imaging requires that the mathematical approach have isotropic sources, which are ideally placed one scattering distance inside the imaging domain. This approach has been well studied to match the classic diffusion assumptions and provide a match to light transport in turbid media over long distances [1]. Poor source placement can lead to image artifacts near the boundary which can lead to inaccurate diagnostic information. NIRFAST is an FEM-based software package designed and freely distributed for modeling Near Infrared Frequency domain light transport in tissue [2]. In most simulations, placement of the source is not an issue, however in real practice, the automated or even semi-automated placement of source locations, corresponding to where fibers are, can be challenging. This is especially important when irregular meshes are created from prior image information, such as with MRI, CT or ultrasound. This study takes a focused look at defining a robust way to place these which will work with irregular domains and models. The source location movement should be inwards along the surface normal direction, yet this is not always possible if the discretization is imprecise near the boundary, or if the fiber is actually at an angle to the surface normal.

In most real 3D tissue meshes, placing the sources and moving them in one mean free transport scattering distance inside the medium can be a challenge. Even with fiducial markers, the orientations of the fibers on the surface are not known. Without these vectors, it is desirable to have an automated method for moving the sources, although admittedly the fiber direction is critical to accurate placement. The methods used previously have been center-based, normal-based, and spiral-search algorithms. Center-based methods choose either the geometric center or the midpoint of the imaging domain, and move the sources on a line towards that point. These are unreliable for non-convex domains, slab geometries, and domains where the sources are off-plane from the center point. The normal-based method finds the closest points on the surface of the domain, and moves the sources orthogonally inwards. This method is also unreliable for non-convex domains since it is possible for the search algorithm to cross external boundaries. The spiral-search method spirals outwards from the source location, stopping at the first point that is at least one scattering distance inside the domain. A dense search is then performed at this point. This method is robust for most imaging domains, but the computational time is unreasonably large. These shortcomings provide the motivation for a better algorithm, as is proposed here. The solution examined involves shrinking the mesh physically, and using the direction from the original mesh to the shrunken mesh as a stable way to find the direction of the inward normal.

\section{Methods}

This surface-shrinking algorithm is described based upon the mesh, consisting of triangles or tetrahedrons for 2D and 3D respectively. This mesh is interpolated onto a dense grid of pixels, each pixel being a very small square or cube. Pixel size is related to the scattering coefficient of the domain. The pixels are labeled as either internal (1) or external (0) to the domain. The pixel-mesh is then shrunk by 
passing over groups of 4 pixels in 2D (forming a square) or 8 pixels in 3D (forming a cube), using rules such as those shown in Figure 1.

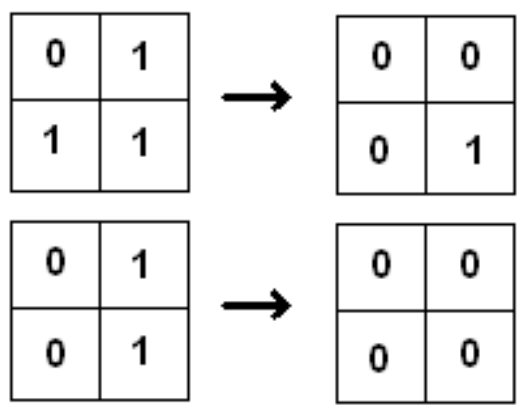

Figure 1: Example rules for shrinking a pixel-mesh in 2D. The fact that there is a 0 on the left hand side indicates that there is a boundary between external (0) and internal (1) nodes. The group of 4 pixels on the left is replaced by the group of 4 pixels on the right, thus shrinking the number of pixels internal to the domain.

The pixel-mesh is passed over $\mathrm{D}_{\mathrm{s}} / \mathrm{w}_{\mathrm{p}}$ times in this manner, where $\mathrm{D}_{\mathrm{s}}$ is the scattering distance and $\mathrm{w}_{\mathrm{p}}$ is the width of each pixel. Thus the mesh has been shrunk by one scattering distance. Note that this is not a geometric contraction, but rather a shrinking of the volume or area towards the kernel. For each source location, the source is moved to the closest point on the pixel-mesh surface.

Another way to shrink the mesh involves moving boundary surfaces inwards orthogonally, and then correcting for intersections of the resulting surfaces. This method is faster than using a pixel-mesh, but correcting for intersections is not trivial. Knowing which side of an intersection to remove involves tracking the nodes back to their original positions.

\section{Results}

The surface-shrinking algorithm was employed on a 3D mouse head mesh [3]. The center of the mesh is not near the planes of the sources, so that the center-based method moves them at a sharp angle to the surface. Figure 2 shows the results of the surface-shrinking algorithm from two different angles. The sources are moved in one scattering distance, and the vectors of movement are orthogonal to the surface.
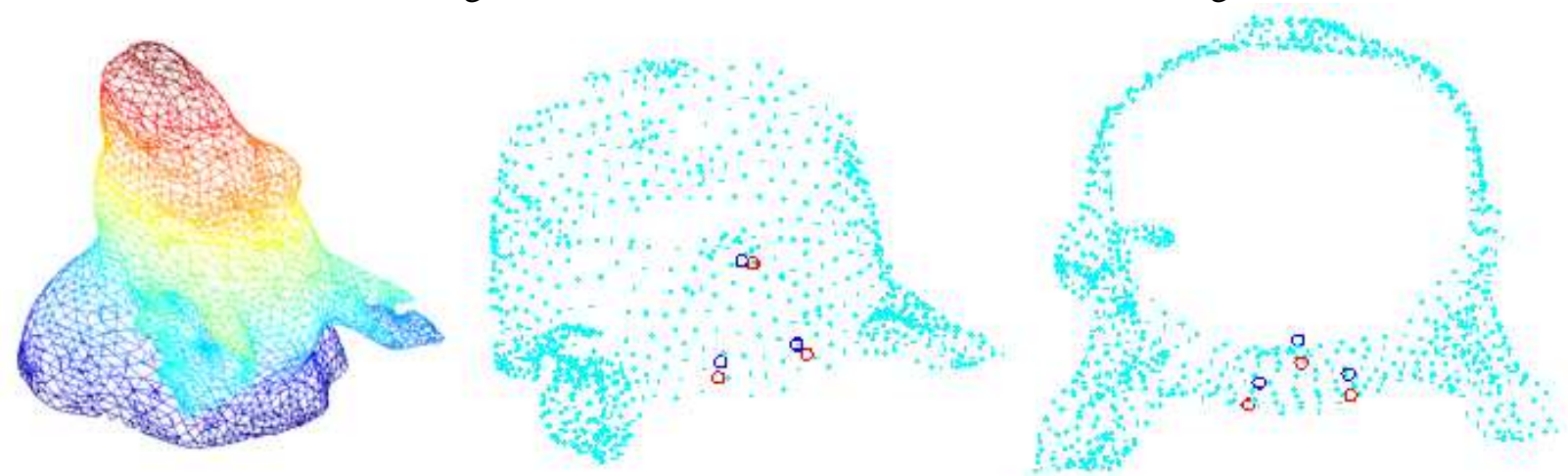

Figure 2: Results of the surface-shrinking method on a 3D mouse head mesh. Only a portion of the surface mesh is shown for better visualization of the source positions. The full surface mesh is shown on the left. The red circles indicate the original source positions, just outside the surface of the mesh. The blue circles indicate the source positions after the being moved by the surface-shrinking method.

Figure 3 shows the results of the surface-shrinking algorithm on a $2 \mathrm{D}$ mesh concocted to thwart the normal-based method. The small irregularity at the top-right corner of the mesh causes the normal-based method to move the nearby source outside of the mesh, whereas the surface-shrinking method places it correctly inside the mesh. Furthermore, center-based methods would move the center source diagonally to the surface. The surface-shrinking method correctly moves it orthogonally. 


\section{BSuD30.pdf}

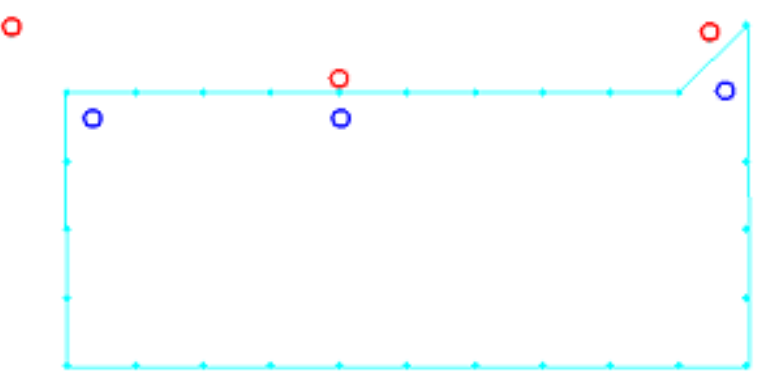

Figure 3: Results of the surface-shrinking method on a 2D custom mesh. Only the surface of the mesh is shown. The red circles indicate the original source positions. The blue circles indicate the source positions after being moved by the surface-shrinking method.

\section{Conclusions}

The ideal method for source movement is to use known vectors for the orientation of the fibers on the surface of domain. Barring the knowledge of these vectors, it makes sense to move in orthogonally, as long as there are no irregularities on the surface that would skew local parameters such as the surface normal. The surface-shrinking algorithm is robust to these irregularities in the imaging domain. This is because the movement of the sources is based on the actual area or volume one scattering distance inside the domain, rather than the local parameters. The algorithm also works when the original source locations are well within the domain or well outside the domain, since the sources are moved to the closest points on the shrunken surface. The computational time of this algorithm is on the order of seconds for any mesh with less than 100,000 nodes on a standard personal computer. Choosing the size of the pixels in the pixel-mesh determines the computational time and accuracy of the process. A smaller pixel size will be more accurate, but increase the computational time.

\section{Acknowledgements}

This work has been funded by NIH grant RO1CA132750.

\section{References}

[1] H Dehghani, M E Eames, P K Yalavarthy, S C Davis, S Srinivasan, C M Carpenter, B W Pogue, K D Paulsen, Near Infrared Optical

Tomography using NIRFAST: Algorithms for Numerical Model and Image Reconstruction Algorithms. Communications in Numerical Methods in Engineering, 2008.

\section{[2] NIRFAST. Dartmouth College, 2009. 〈http://www.nirfast.org>}

[3] S L Gibbs-Strauss, J A O’Hara, S Srinivasan, J P Hoopes, T Hassan, B W Pogue, Diagnostic detection of diffuse glioma tumors in vivo with molecular fluorescent probe-based transmission spectroscopy. Medical Physics, volume 36, 2009. 